\title{
Investigation of Enzymatic Degradation of Solid Winemaking Wastes
}

\author{
Galyna Krusir', Olga Sagdeeva*1, Myroslav Malovanyy ${ }^{2}$, \\ Hanna Shunko', Oleksandr Gnizdovskyi ${ }^{3}$
}

1 Department of Ecology and Environmental Technologies, Odessa National Academy of Food Technologies, str. Kanatnaya, 112, Odessa, 65039, Ukraine

2 Viacheslav Chornovil Institute of Sustainable Development, Lviv Polytechnic National University, S. Bandera Str. 12, Lviv, 79013, Ukraine

3 LLC "SC "Expert Center", Narodnogo Opolchennia Str., 1, of. 319, Kyiv, 03151, Ukraine

* Corresponding author's e-mail: sagolanis@ukr.net

\begin{abstract}
The work is devoted to the research of the enzymatic destruction process of solid waste of the primary winemaking enterprises the introduction of advanced technology of their utilization and creation of optimally balanced feed additive. The processing of grapes produces waste, the storage and accumulation of which in environmental components leads to the loss of valuable resource in the composition of waste and leads to the creation of increased levels of environmental hazards. Enzymatic degradation of cellulose in the composition of waste by the cellulase enzyme through the microorganisms' action is the basis of the biotechnological process. To implement this process, it is necessary to determine the main parameters and modes of the bioconversion process, investigation of which the study is devoted. The basic characteristics of the enzymatic degradation process were determined and data were obtained for the technology improvement of solid waste utilization of the wine industry enterprises. Comparative analysis of bioconversion of different types of waste proves that the cellulose enzymatic degradation is the most effective for the grape pomace in the waste, allows us to obtain valuable feed additive and reduce the environmental danger level.
\end{abstract}

Keywords: winemaking waste, grape extract, enzymatic degradation, bioconversion, cellulose, cellulase, environmental safety

\section{INTRODUCTION}

The waste accumulation and management is the first among the environmental problems in Ukraine. The organic waste management technologies of the national economy objects, in particular, food enterprises, have been received a great deal of attention both nationally and internationally, due to their specificity and extent of formation and relative homogeneity.

Thus, as a result of the activity of the primary winemaking enterprises, together with discharges of concentrated sewage into the hydrosphere and emissions into the atmosphere, is the annual formation of over 80 thousand tons of solid wastes. Such solid wastes have a wide nomenclature and specific composition and contribute to the formation of environmental dangers in the case of their placement in environmental components.

When processing grapes for grape must, wine fermentation, processing and distilling wine materials, waste is formed, which includes such valuable components as sugar, alcohol and tartaric compounds. The waste such as grape pomace, stalks, yeast deposits, distillery cognac dregs and tartar are recyclable to obtain useful components. That allows obtaining food and feed products for a number of the economy sectors, as well as reducing the environmental burden.

Scientific researches (Kostylev, 2009) in the field of the secondary wine raw materials recycling contain a number of pilot-experimental and analytical works (Krusir et al., 2013). As a result of the industrial processing of grapes, a 
significant amount of waste is generated, which accounts for 10 to $20 \%$ of the amount of raw material being processed. The total potential of food and feed by-products of winemaking waste can be estimated by the methods of their utilization (Fig. 1) (Krusir et al., 2014).

\section{PROBLEM FORMULATION}

Bioconversion of renewable plant materials into fuels, feed and food, intermediates for the chemical and microbiological industries is now considered as one of the key biotechnology industries.

Grapes by content of useful components are one of the richest representatives of perennial cultivated plants. A significant part of the grape components after its industrial processing remains in the secondary products - winemaking waste, the main types of which are the grape pomace, stalks, yeast deposits and wine stone. However, in terms of the formation volumes the grape pomace prevails over all other types of the winery solid waste, so one of the most urgent solutions to the problem of winemaking waste recycling is the processing of the grape pomace to feed additive.

The grape pomace includes the grape stalks, husks and seeds, which are both separate wastes of winemaking and a component of the pomace mix. The results of the chemical composition analysis of the grape pomace (Table 1) indicate the value of raw materials for the production of feed additives for cattle by the content of lipids, proteins, carbohydrates, nitrogenous and other compounds

Among the nutrients of the grape pomace a protein has a great importance; its content is $15.0 \%$. The biological value of a protein depends on its amino acid composition (Gutsulak, 1992), so the next stage of the study was to study the amino acid composition of the grape pomace, which revealed that the grape pomace is rich in critical amino acids such as tryptophan (7.2\%) and lysine $(7.5 \%)$, the presence of which contributes to weight gain and optimal metabolism in animals (Kalachnyuk et al., 1986, Sakhno, 1995).

As mentioned above, the grape pomace contains fragments of the grape husks and seeds in a ratio of 2:1, so one of the most important steps in the research was the detailed study of the chemical composition of both grape husks and grape seeds included into the grape pomace.

Lipids, being isolated from grape seeds, contain $87.0-93.0 \%$ of physiologically valuable unsaturated fatty acids, including linoleic (1.3\%), linolenic (1.1\%), oleic (3.4\%), arachidonic acids $(19.0 \%)$, which are the main source of energy reserve material and perform extremely important physiological functions in cell metabolism and in enzymatic processes (Krusir et al., 2012.).

According to the results of experimental studies, the grape husks are rich in carbohydrates, cellulose $(39.0 \%)$, but poor in lipids $(0.4 \%)$ and protein $(5.8 \%)$.

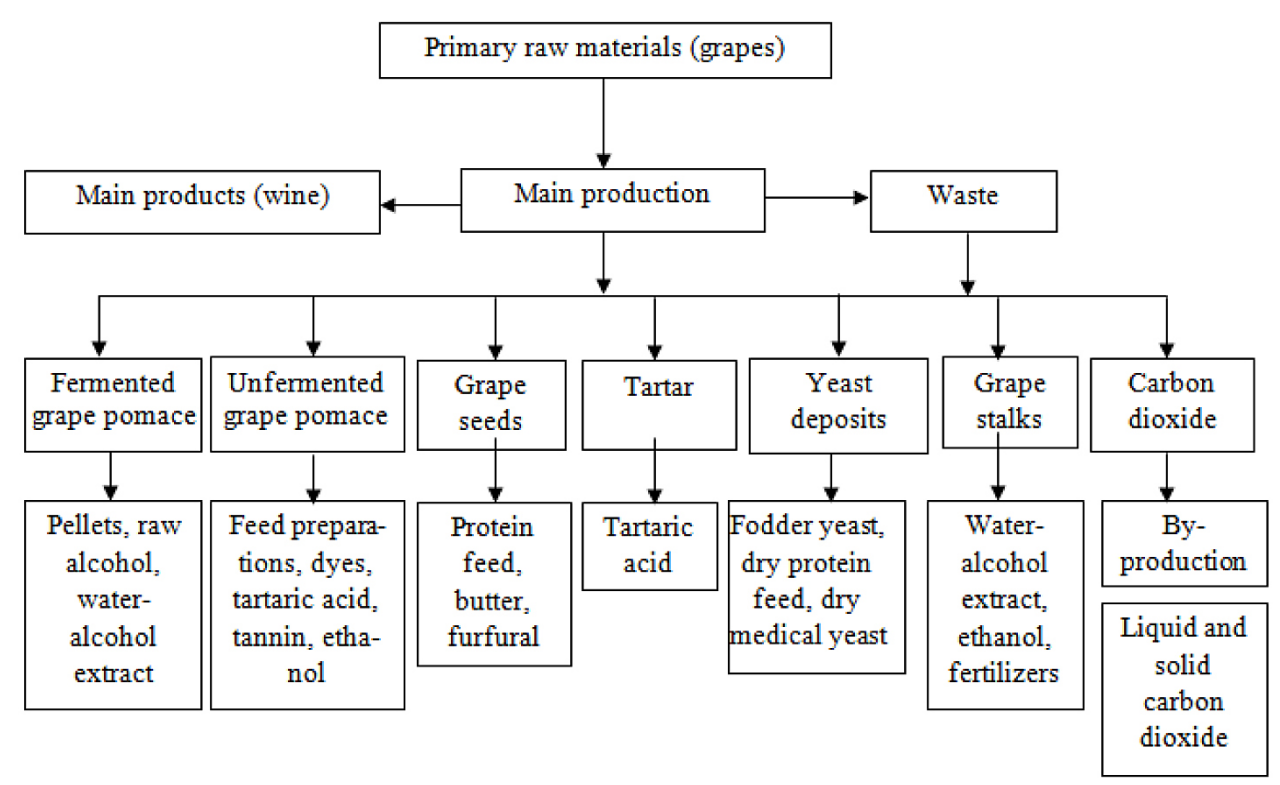

Fig. 1. Scheme of the utilization methods of the wineries production waste 
Table 1. The grape pomace chemical composition

\begin{tabular}{|c|l|c|}
\hline No. & Group of substances & $\begin{array}{c}\text { \% in terms of dry } \\
\text { matter }\end{array}$ \\
\hline 1 & Lipids & 9.0 \\
\hline 2 & $\begin{array}{l}\text { Hydrocarbons, } \\
\text { including: }\end{array}$ & 20.1 \\
\hline 3 & $\bullet$ Monosaccharides & 28.0 \\
\hline 4 & • Cellulose & 36.1 \\
\hline 5 & Lignin & 19.0 \\
\hline 6 & Protein (total) & 15.0 \\
\hline 7 & Ash & 1.5 \\
\hline 8 & Phenolic compounds & 6.0 \\
\hline
\end{tabular}

Thus, the predominant substance of the grape pomace chemical composition is carbohydrates, namely, cellulose $(36.1 \%)$, which must be degraded in the solid waste compound. Cellulose refers to polymers that are poorly soluble, it is determined by the crystalline structure and the cellulose chains packing into microfibrils.

The chemical properties of cellulose are determined by the presence of a $\beta$-glycosidic linkage and by three hydroxyl groups in each glucose residue. Amorphous areas of cellulose react faster than crystalline ones. Dense packing of cellulose fiber generally prevents the attack with reagents, resulting in cellulose is characterized by largely toponymic (surface) reactions. That is why enzymatic hydrolysis is essential for the destruction of cellulose, which is the basis of grape pomace, however the cellulose is subjected to chemical action or grinding before the process.

The aim of the research is to study the parameters and the course of the processes of enzymatic degradation of winemaking wastes, in particular the grape pomace, for improving their utilization process and creating an optimally balanced feed additive.

\section{INVESTIGATION OF ENZYME PROPERTIES}

Enzymatic transformation of cellulose is promising not only from point of view of separate low-waste technologies creation, but also from point of view of the ecological danger reduction of various productions, which process vegetable raw materials and are accompanied by the formation of a considerable amount of waste.

The cellulase enzyme catalyzes the breakdown of cellulose to glucose, cellobiose and oligosaccharides production. It is synthesized by microorganisms (bacteria, microscopic fungi and actinomycetes), which mainly hydrolyze cellulose and do not use other components of the nutrient medium as energy sources.

Cellulases are obtained by deep or more often surface cultivation of microorganisms on media with different cellulose-containing substrates (bran, exhausted cossettes, sawdust, straw, woody pulp, paper and viscose mass), purified cellulose, molasses and simple sugars. The enzyme complex formation is influenced by the composition and ratio of the components, the acidity and aeration of the medium. Of the currently known cellulase producers, the most active enzymes producers that are capable of saccharifying natural cellulose-containing substrates are various strains of fungi of the genus Trichoderma. The advantage of Trichoderma fungi, as a cellulase source, is that they produce a sufficiently complete complex of high yield cellulases. Trichoderma fungi were found to exhibit three types of cellulasolytic activity: exo- $\beta-1,4$-glucanase (KF.3.2.1), endo$\beta$-1,4-glucanase (KF.3.2.1.4) and $\beta$-glucosidase (KF.3.2.1.21).

To determine the features of enzymatic degradation and to determine the optimal regimes of the process, it is necessary to study the physicochemical properties of cellulase. The $\mathrm{pH}$ optimum of cellulolytic enzymes was found to be in the range of 4 to $6 \mathrm{pH}$ units. Cellulolytic enzymes are characterized by relatively high thermal stability, the temperature optimum of many of them is $60^{\circ} \mathrm{C}$. The qualitative and quantitative composition of the cellulose complex depends on the type of producer and the conditions of its cultivation.

In the first study stage, the exoglucanase, endoglucanase and glucosidase activities of the Trichoderma cellulolytic complex were determined to be $880,109.1$, and $112.9 \mathrm{u} / \mathrm{mg}$ cellulase, respectively. The results of the $\mathrm{pH}-$ and thermooptimum and enzyme stability studies are presented in Figures 2-4.

From the presented in Fig. 2 experimental data we can conclude that the $\mathrm{pH}$-optimum of cellulase is observed in the region of 5.0, which indicates that the enzyme belongs to the group of acidic cellulases, which is further indicate a sharp decrease in the enzyme activity with increasing $\mathrm{pH}(6.0)$ and complete lack of activity when $\mathrm{pH}=10.0$.

When carrying out an enzymatic reaction, there are two different factors, that deal simultaneously, determining the temperature effect: on the one hand - an increase in the initial rate, on 


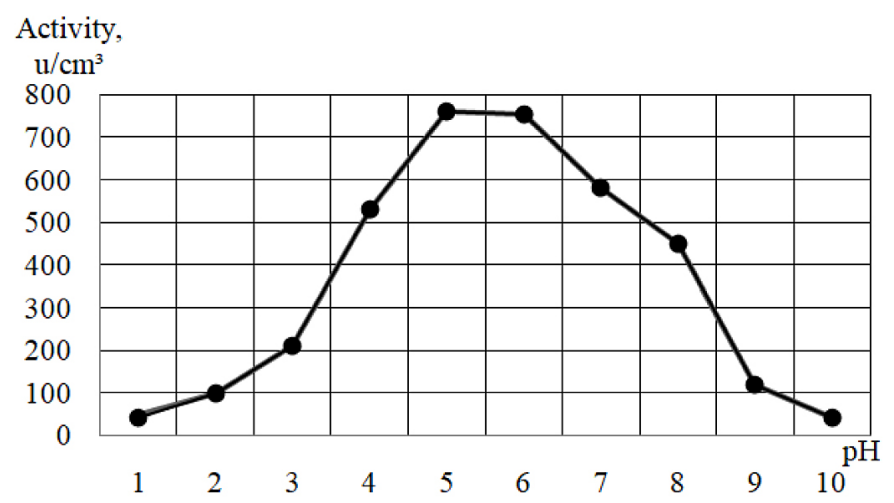

Fig. 2. pH-optimum of the cellulose enzyme

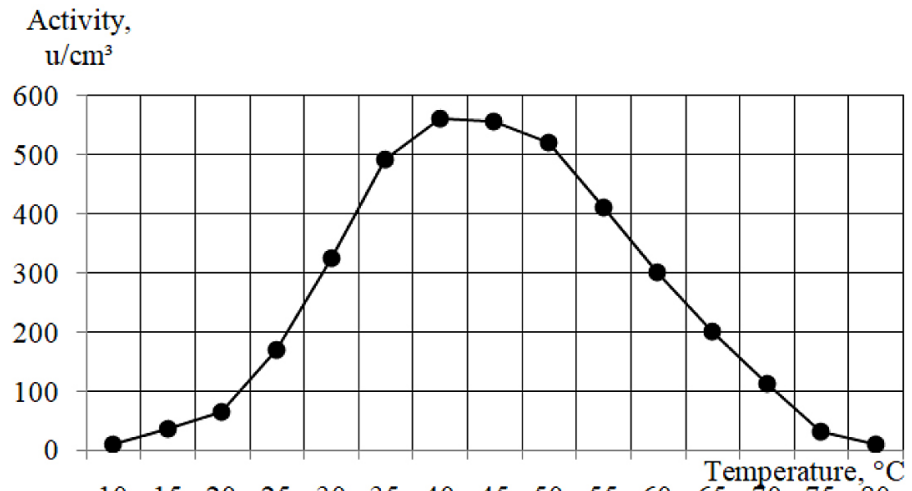

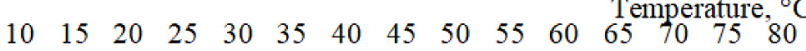

Fig. 3. Thermo-optimum of the cellulase enzyme

the other - denaturation of the enzyme under the action of temperature, which causes a continuous decrease in the concentration of the active enzyme. The optimal temperature depends on the relationship between the temperature effect on the rate of the enzymatic reaction and its effect on the rate of the enzyme denaturation.

The thermo-optimum of the cellulase enzyme was determined at the optimum $\mathrm{pH}$ value (5.0) and the temperature change in the range from 20 to $80^{\circ} \mathrm{C}$. The maximum activity of the enzyme (Fig. 3) is observed in the temperature range of $40-45^{\circ} \mathrm{C}$, with its rapid decrease with further increase in temperature, which is caused by thermal denaturation of the native protein molecule.

pH stability (Fig. 4) was investigated at the optimum $\mathrm{pH}$ value (5.0 $\mathrm{pH}$ units), in acidic medium at $\mathrm{pH}<6.0$ and in alkaline medium at $\mathrm{pH}>8.0$

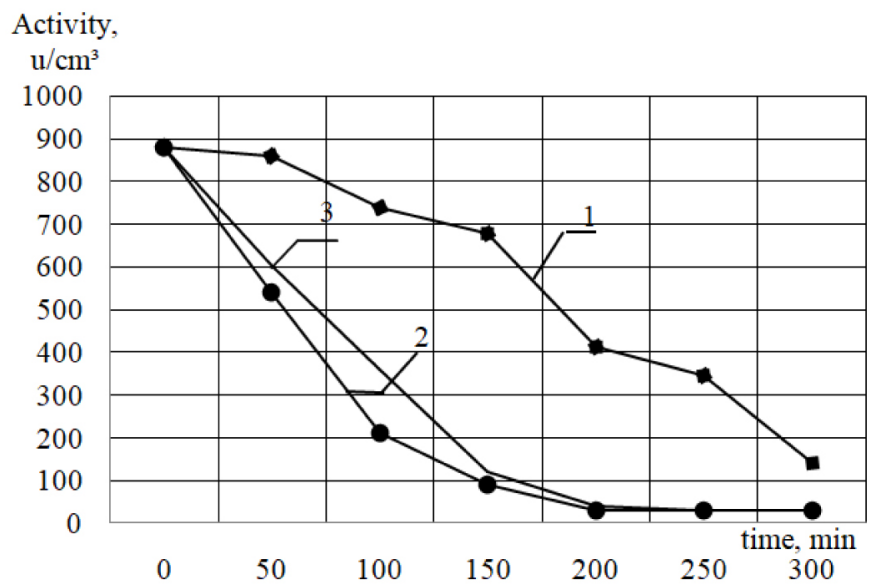

Fig. 4. $\mathrm{pH}$ stability of the cellulase enzyme at $40^{\circ} \mathrm{C}$, where $1-\mathrm{pH}=5 ; 2-\mathrm{pH}=2.5 ; 3-\mathrm{pH}=8$ 
at $40^{\circ} \mathrm{C}$ for 6 hours. In acidic environments, the activity decreased sharply during the first 20 minutes. At alkaline $\mathrm{pH}$ values, a slower inactivation of the enzyme was observed. Complete loss of activity was observed after 3 hours of the enzyme incubation. Under optimal conditions of enzyme functioning the activity is stable for 50 minutes, and then it decreases and is completely lost after 4 hours due to protein denaturation.

The temperature effect on the rate of enzymatic reactions may be due to the action of various factors. Temperature affects the enzyme stability, the decay rate of the enzyme substrate complex, the affinity of the enzyme with the substrate. The cellulase thermal stability was determined at $\mathrm{pH}=5.0$ and at temperature values of 20 , 40, 60 and $80^{\circ} \mathrm{C}$ (Fig. 5).

The obtained results (Fig. 5) allow us to find out that the maximum activity of the enzyme was observed at a temperature of $40^{\circ} \mathrm{C}$, complete loss of activity occurred after 200 minutes. At a temperature of $60^{\circ} \mathrm{C}$ and above there was a sharp decrease in the rate of hydrolysis reaction due to thermal denaturation of the protein.

\section{THE EXPERIMENTAL PART}

The purpose of this work stage is to determine the most optimal modes of enzymatic hydrolysis of the grape pomace, for which the role of such parameters as the temperature influence $\left(\mathrm{T},{ }^{\circ} \mathrm{C}\right)$, water duty (WD), the duration of the enzymatic hydrolysis process, $(\tau, \mathrm{min})$, the $\mathrm{pH}$ of the reaction medium, the grinding degree and the enzyme solution concentration on the process of fermentolysis were investigated (Sokolova IF. et al., 2014).
From the literature analysis it is known that one of the important parameters of the cellulose hydrolysis process is the water duty, so the next step was to study the effect of WD, shown in Fig. 6. The obtained results of the study, presented in Fig. 6, allow us to determine that the maximum degree of hydrolysis for cellulose for the grape pomace is the highest at WD 9 and it is $67.1 \%$. The WD increasing does not lead to a significant increase in the hydrolysis level, so it is not advisable to increase the WD.

There are various methods of pre-treatment of cellulose-containing raw materials in order to increase the hydrolysis degree. The mechanical pretreatment method involves the grinding of raw materials. The influence of the substrate grinding degree on the hydrolysis degree is shown in Fig. 7.

The change in the hydrolysis degree from the particle size, shown in Fig. 7, allows us to conclude that the maximum degree of cellulose hydrolysis is achieved with a particle size of $500 \mu \mathrm{m}$. This is because the grinding increases the contact surface area of the biopolymers of the raw material with the enzyme complex and leads to an increase in the rate of enzymatic transformations.

One of the most important factors that influence the fermentation process is the temperature regime. The temperature effect on the cellulose hydrolysis process is shown in Fig. 8.

Thus, the data of Fig. 8 determine that the maximum of the cellulose hydrolysis degree is observed at a temperature of $40^{\circ} \mathrm{C}$ for the grape pomace and it is $70.1 \%$. With increasing temperature, the degree of hydrolysis decreases due to the thermal denaturation of the enzyme protein part.

The next stage of research was to determine the optimal $\mathrm{pH}$ value for the enzymatic hydrolysis

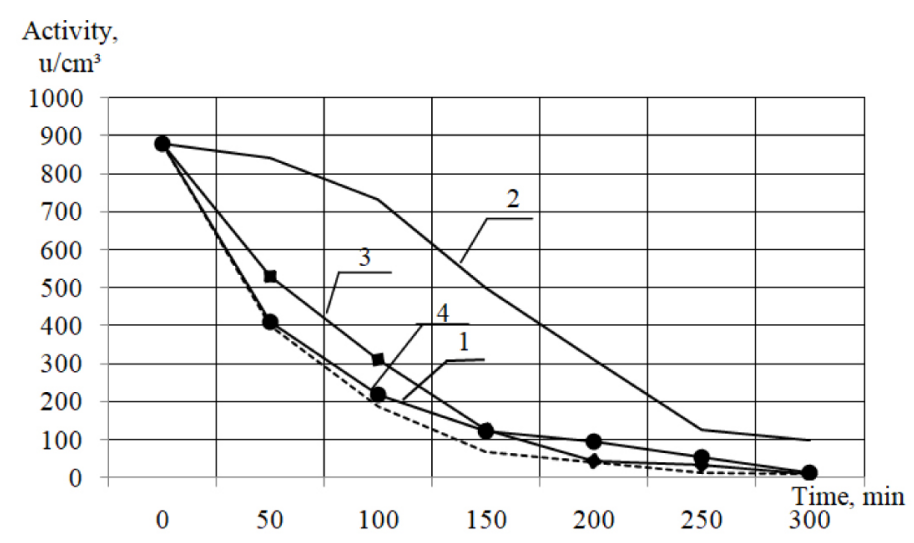

Fig. 5. Thermostability of the cellulase enzyme at $\mathrm{pH}=5.0$, where $1-20^{\circ} \mathrm{C}, 2-40^{\circ} \mathrm{C}, 3-60^{\circ} \mathrm{C}, 4-80^{\circ} \mathrm{C}$. 


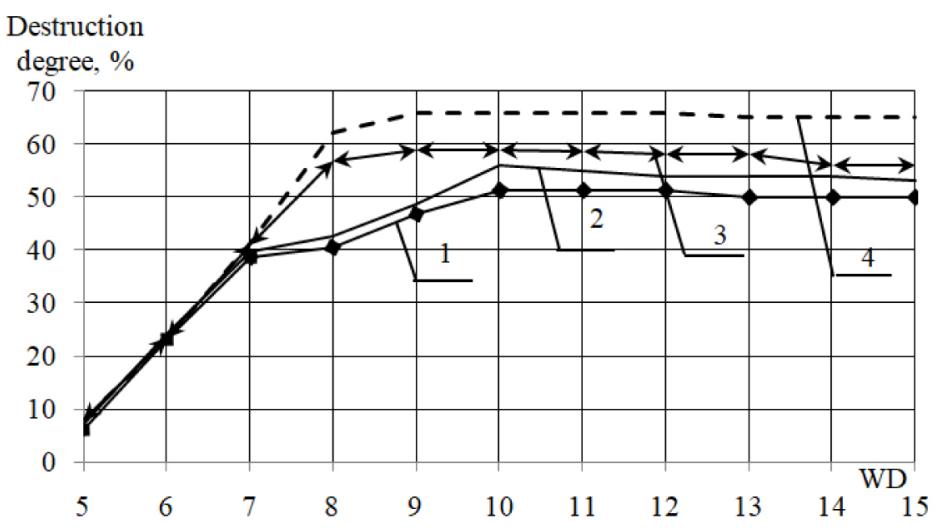

Fig. 6. Dependence of the cellulose hydrolysis degree (\%) on WD,

where 1 - the grape seeds, 2 - the grape stalks, 3 - the grape husks, 4 - the grape pomace

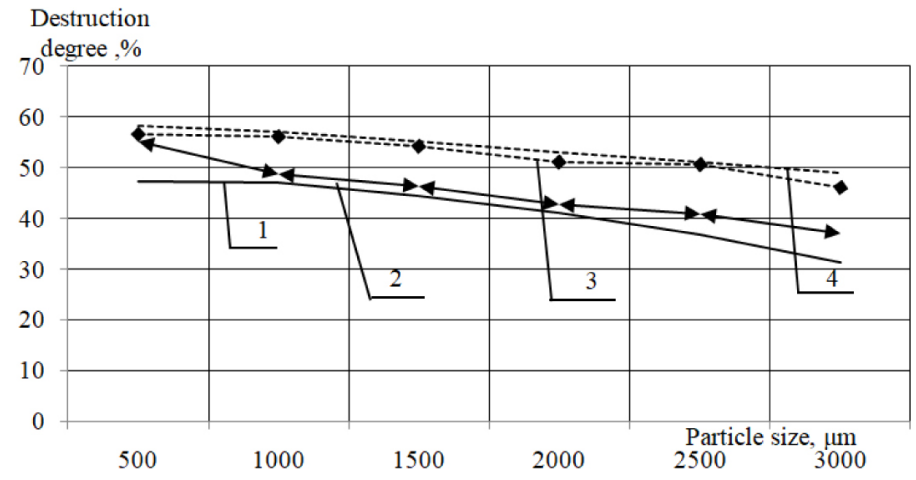

Fig. 7. Dependence of the cellulose hydrolysis degree (\%) on the substrate grinding degree, where 1 - the grape seeds, 2 - the grape husks, 3 - the grape stalks, 4 - the grape pomace

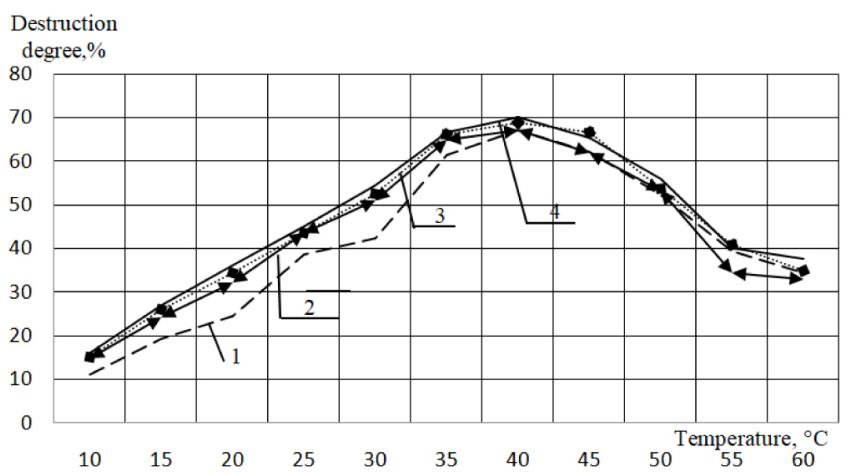

Fig. 8. Dependence of the cellulose hydrolysis degree (\%) on temperature,

where 1 - the grape seeds, 2 - the grape stalks, 3 -the grape husks, 4 - the grape pomace

process of cellulose (Fig. 9), since the enzymes activity depends on the $\mathrm{pH}$ of the solution in which the enzymatic reaction takes place.

The data, presented in Fig. 9, allow us to determine that the maximum degree of cellulose hydrolysis in the substrate is observed at $\mathrm{pH}=5.0$ exactly for the grape pomace and it is $56.2 \%$.
The process duration of enzymatic hydrolysis is essential for improving the technology of waste processing into the feed resource, so the effect of the process duration on the degree of cellulose destruction was determined separately (Fig. 10).

The study of dependencies in Fig. 10 lead to the conclusion that the destruction of the grape 


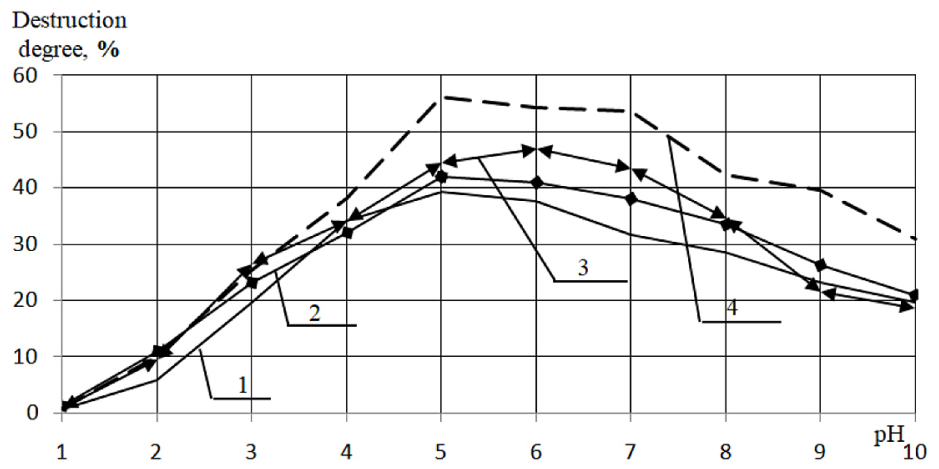

Fig. 9. Dependence of the cellulose hydrolysis degree (\%) on the $\mathrm{pH}$ value of the medium where 1 -the grape seeds, 2 - the grape husks, 3 - the grape stalks, 4 - the grape pomace

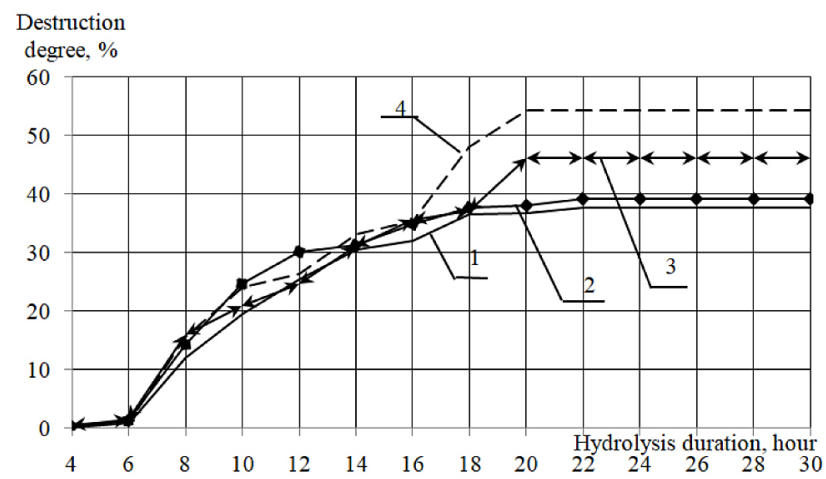

Fig. 10. Dependence of the cellulose hydrolysis degree (\%) on the fermentation process duration where 1 - the grape seeds, 2 - the grape husks, 3 - the grape stalks, 4 - the grape pomace

pomace lasts the least time compared to other types of waste, taken separately, and it takes 20 hours with a degradation rate of $54.3 \%$ versus 22 hours and destruction of the grape seeds and husks up to $40 \%$. Further retention of the substrate is impractical.

The enzyme concentration significantly influences the speed of enzymatic reaction and cellulose destruction (Sakhno OV., 1995, Sokolova IF. et al., 2014), so determining the optimal concentration of cellulase is an important stage of research and it is presented in Fig. 11.

Data of Fig. 11 allow us to conclude that the highest degree of cellulose destruction for the grape pomace in fermentation by cellulose is observed at an enzyme concentration of $0.3 \%$ and it

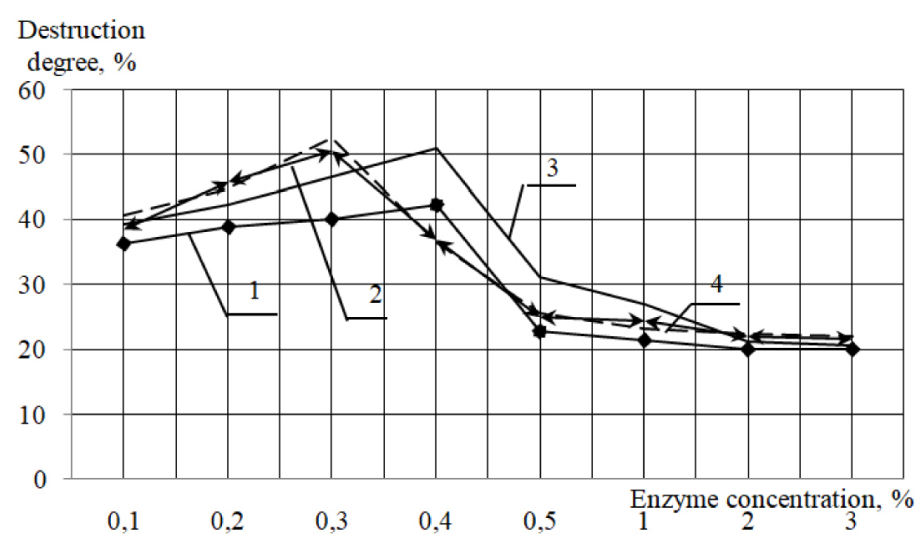

Fig. 11. Dependence of the cellulose hydrolysis degree (\%) on the concentration of the enzymatic solution where 1 - the grape seeds, 2 - the grape husks, 3 - the grape stalks, 4 - the grape pomace 
is the maximum value (52.6\%). Increasing the enzyme concentration does not lead to a significant increase in the destruction degree, and a decrease in the concentration leads to its decrease (Sokolova IF. et al., 2014).

\section{CONCLUSIONS}

1. Investigations into the enzymatic degradation processes of winemaking waste have made it possible to determine the basic physicochemical properties of the cellulose enzyme that affect the destruction process, and hence the waste processing, and the optimal regimes of the fermentation process.

2. Thus, it is found that the $\mathrm{pH}$ optimum of cellulase is in the region of $5 \mathrm{pH}$ units, and the $\mathrm{pH}$ stability is observed for 50 minutes. The maximum activity of the enzyme was observed at a temperature of $40^{\circ} \mathrm{C}$, and its complete loss occurred after $200 \mathrm{~min}$.

3. The most optimal parameters of the enzymatic hydrolysis process of the grape pomace were obtained in comparison with the destruction of certain types of waste, such as the grape seeds, husks and stalks of grapes. Comparative analysis leads to the conclusion that enzymatic degradation is of paramount importance for the grape pomace, since its degree is maximum for the complex substrate, as studies have shown.

4. Thus, the maximum degree of hydrolysis for cellulose for the grape pomace is greatest at WD $9(67.1 \%)$, at a temperature value of $40^{\circ} \mathrm{C}$ $(70.1 \%)$ and at a $\mathrm{pH}$ value of 5.0 units $(56.2 \%)$. Also, the destruction of the grape pomace lasts the least time compared to other types of waste and takes 20 hours at a degradation rate of $54.3 \%$, and the highest degree of cellulose destruction for the grape pomace by the cellulose fermentation is observed at an enzyme concentration of $0.3 \%$ and it is the maximum value $-52.6 \%$.
That's why the enzymatic degradation of the grape pomace, as a common waste of the food industry, in particular, the wine industry, is a relevant and effective biotechnological processing method to produce a useful product (feed additive), to increase the role of resource conservation and to reduce the environmental safety level by reducing solid waste.

\section{REFERENCES}

1. Gutsulak VD. 1992. Bioconversion of organic waste to produce biohumus, biogas, biological substances and environmental protection. Plant protection, 1, $61 \mathrm{p}$.

2. Kalachnyuk GN. et al. 1986. Recommendations on the use of alternative feeds for fattening young cattle. Kiev, 268 p.

3. Kostylev NV. 2009. Identification of objects and sources of negative environmental impact. Perm, $57-59$.

4. Krusir GV. et al. 2012. Solid waste - ecological aspects of winemaking enterprise. Environmental safety, Kremenchuk, 2/2012 (14), 112 - 115.

5. Krusir GV. et al. 2013. Index assessment of the environmental hazard of wineries. Ecological safety, Kremenchuk, 1/2013 (15), 96 - 98.

6. Krusir GV. et al. 2014. Features of the use of index indicators in the assessment of the level of environmental hazard of industrial enterprises. International scientific conference dedicated to the 130th anniversary of the National University of Food Technologies "New ideas in food science - new products of the food industry", Kiev, 717 p.

7. Sakhno OV. 1995. The influence of bioconverse conversion of grapes for productivity and the exchange of grains for pigs for annual growth. Abstract of diss. Ph.D. H., 25 p.

8. Sokolova IF. et al. 2014. Investigation of the chemical composition of solid wastes of primary winemakingt. XIV All-Ukrainian Scientific and Technical Conference of Young Scientists and Students "Ecological and Energy Problems of Modernity", Odessa, $49-51$. 